\title{
SATISFAÇÃO DO CONSUMIDOR: ESTUDO BIBLIOMÉTRICO EM UMA BASE DE DADOS NACIONAL
}

\author{
CONSUMER SATISFACTION: \\ A BIBLIOMETRIC STUDY IN A NATIONAL DATA BASE
}

Franciely Buehrmann $^{1}$ e Nathalia Berger Werlang ${ }^{2}$

Recebido em: 10/07/2016 Aprovado em: 09/01/2017

\section{RESUMO}

Este estudo tem como objetivo identificar as principais tendências em pesquisas sobre o tema satisfação do consumidor a partir de um mapeamento bibliométrico em uma base de dados nacional. $\mathrm{O}$ método empregado foi a bibliometria, utilizandose uma amostra de 60 artigos. Para facilitar a visualização dos resultados, os dados foram tratados com o software excel e interpretados pela análise de conteúdo. Os trabalhos analisados compreenderam o período de 2010 a 2015. Os resultados sugerem que: o tema é de crescente interesse no meio acadêmico nos últimos anos; a Universidade de Fortaleza é a Instituição de Ensino Superior (IES) de destaque na produção científica acerca da satisfação do consumidor; dentre os periódicos que mais publicam estudos sobre o tema, destaca-se a Revista Brasileira de Marketing; os anos de 2012, 2013 e 2014 sobressaíram-se pelo número de publicações sobre satisfação do consumidor; a escala de medida elaborada por Oliver (1997) é a mais utilizada para mensuração deste construto; pesquisas quantitativas do tipo survey destacam-se dos estudos qualitativos ou mistos; os autores afirmam que o tema satisfação do consumidor é revolucionário e ainda merece ser desenvolvido. Por fim, identificou-se a importância do desenvolvimento de futuras pesquisas sobre o tema em outras empresas, de diferentes setores, principalmente na indústria e também em micro e pequenas empresas.

Palavras-chave: Comportamento do Consumidor. Satisfação do Consumidor. Bibliometria.

\begin{abstract}
This study has the aim of identifying the main tendencies in researches about the consumer satisfaction issue, from the bibliometric mapping on a base of national data. The applied method was a bibliometric, using a sample of 60 (sixty) articles. To facilitate the view of the results, data were dealt with software excel, being interpreted by the analyses of its content. The analyzed work reached the period from 2010 to 2015. The results suggest that: the theme is of increasing interest in the academy the latest years; the university of Fortaleza is the High Education Institution that outstands in the scientific production of consumer satisfaction; among the journals that publish the most about the theme - Revista Brasileira de Marketing - is outstanding; the years of 2012, 2013 e 2014 outstand for the number of publications on consumer satisfaction, the scale of measurement elaborated by Oliver (1997) is the most used for the measurement of this construct; quantitative researches of the survey type outstand the qualitative and hybrid studies, the authors claim that the theme consumer satisfaction is revolutionary and still deserves to be developed. Finally, the importance of the development of future researches on the theme, in different sectors, mainly in industry, as well as in micro and small businesses, was identified. Keywords: Consumer Behavior. Consumer Satisfaction. Bibliometric.
\end{abstract}

1 Possui graduação em Licenciatura em Matemática pela Universidade Federal de Ouro Preto - UFOP (2006), Mestrado (2010) e Doutorado (2014) em Demografia pelo Centro de Desenvolvimento e Planejamento Regional - CEDEPLAR da Universidade Federal de Minas Gerais - UFMG (2010) e Pós-doutorado na área de Economia Doméstica pela Universidade Federal de Viçosa - UFV (2015). Atualmente realiza o segundo pós-doutorado também na área de Economia Doméstica pela Universidade Federal de Viçosa - UFV.

2 Doutor em Demografia pelo Centro de Desenvolvimento e Planejamento Regional CEDEPLAR da Universidade Federal de Minas Gerais. Graduação em Ciências Econômicas pela Universidade Federal de Uberlândia - UFU. Atualmente é servidor da UFU, ocupando o cargo de Diretor de Provimento, Acompanhamento e Administração de Carreiras. Pertence ao quadro de pesquisadores do Centro de Pesquisas Econômico-Sociais - CEPES do Instituto de Economia. 


\section{Introdução}

O crescimento das organizações, as mudanças no comportamento dos consumidores e as demandas exigidas pelo consumidor têm transformado o ambiente de negócios cada vez mais competitivo e desafiador. Estes fatores têm obrigado as organizações a realizar um processo de avaliação que exige considerar seu posicionamento de mercado e trabalhar em busca de diferenciais competitivos inertes. Além disso, trabalha-se a partir do estabelecimento de estratégias que envolvam desde o atendimento às necessidades e aos desejos do cliente até a criação e a manutenção de um relacionamento lucrativo para ambos, empresa e cliente (LEITE, FILHO, 2007).

Nesse contexto, a satisfação do consumidor é considerada uma resposta à avaliação da conexão percebida entre a expectativa e a performance após o consumo de um produto/serviço (BLACKWELL et al., 2005). Ou seja, o produto de uma organização pode ser avaliado por diversos públicos - e até mesmo ser copiado pela concorrência. Essas mudanças, por consequência, estão levando as empresas a monitorar o ambiente e pesquisar o mercado continuamente, assim como a escolher cuidadosamente seus clientes e fornecedores com a finalidade de estabelecer relações comerciais duradouras (CONSTANTIN, ABICHT, CEOLIN, 2013). Em decorrência disso, não há como desconsiderar que, ao longo dos anos, os profissionais do marketing precisaram levar em conta com mais ênfase as questões do comportamento do consumidor, a avaliação e a satisfação do consumidor após a compra do produto, como garantia de que o consumidor retornasse à empresa para comprar novamente.

Por isso, torna-se importante e necessário o estudo do comportamento do consumidor; afinal, este é grande relevância para o desenvolvimento de estratégias e de ferramentas mercadológicas em empresas que visam obter vantagens competitivas no atual ambiente dinâmico e globalizado: o mercado.
Dessa forma, o presente estudo tem como objetivo principal identificar as mais significativas tendências em pesquisas sobre o tema satisfação do consumidor a partir de um mapeamento bibliométrico em uma base de dados nacional. Justificam-se novos estudos como essenciais para avançar no tema e obter resultados mais verdadeiros a respeito da satisfação dos consumidores por meio de indicadores técnicos de desempenho e da avaliação dos serviços prestados pelas organizações (MARCHETTI, PRADO, 2004).

Para isso, este estudo foi estruturado em quatro seções, além desta introdutória. Na segunda parte, é apresentado o referencial teórico abordando a conceituação do comportamento do consumidor, a satisfação do consumidor e as principais pesquisas sobre o tema. A terceira parte, por sua vez, contempla o percurso metodológico que fundamenta o artigo. Na sequência, apresentam-se os resultados e, por fim, as considerações mais relevantes acerca da temática abordada, assim como as limitações desta pesquisa e as sugestões para estudos futuros.

\section{Comportamento do consumidor}

O consumidor é o foco de todos os tipos de organizações mercadológicas. Isso evidencia o motivo pelo qual as empresas competem pela conquista e preferência dos consumidores, assim como expõe o esforço que realizam a fim de que o negócio sobreviva. Por isso, as empresas têm dedicado cada vez mais tempo e recursos para entender a dinâmica do processo de tomada de decisões de compra dos consumidores (SAMARA, MORSCH, 2005).

Para Kotler (2000), o estudo sobre o comportamento do consumidor compreende que as pessoas, os grupos e as organizações escolhem, compram, usam e descartam artigos, ideias ou experiências para satisfazer suas necessidades e seus desejos. Ou seja, o estudo do comportamento do consumidor tem 
despertado diversos interesses em diferentes áreas e profissões, como educação, marketing, comunicação, política, direito e proteção do consumidor (SAMARA, MORSCH, 2005).

$\mathrm{O}$ entendimento do comportamento do consumidor não é uma tarefa simples, e sua abrangência é fundamental para os negócios das organizações. Segundo Engel, Blackwel e Miniard (2000), o comportamento do consumidor possui diversas etapas: reconhecimento do problema; busca da informação; avaliação das alternativas geradas (critérios de escolha); e decisão de compra - quando o consumidor compara as necessidades que tinha (suas expectativas com o produto e/ou serviço) e o que recebeu em seu ato de consumo.

Sampaio et al. (2004) afirmam que os consumidores são reconhecidos de diferentes formas, como, por exemplo, por meio de seus costumes, suas culturas e suas personalidades. Isso se torna um fator importante para que os tomadores de decisão, durante este processo, possam estruturar o lançamento e o desenvolvimento de novos produtos no mercado, considerando que as organizações atuais precisam entender da melhor forma possível seus consumidores quanto ao modo de pensar, trabalhar e/ou divertir-se, bem como compreender se são influenciados por outros indivíduos e grupos.

O comportamento do consumidor é composto de influências tanto interpessoais quanto intrapessoais (SAMARA, MORSCH, 2005). Esses autores apresentam um modelo completo dessas variáveis e suas influências: a primeira é a influência dos grupos e atividades no comportamento do consumidor - como a cultura, a subcultura, a família, a classe social e o estilo de vida. A segunda é a análise das forças individuais no comportamento de consumo, tais como: a aprendizagem e experiência, a personalidade, o processo de avaliação, as atitudes, a mudança e os processos de informação. Por último, há a análise do processo de decisão do consumidor, que envolve o reconhecimento do problema, a busca de informações, a avaliação de alternativas, o processo de compra e os comportamentos pós-compra.

Também é preciso considerar que o comportamento do consumidor é influenciado por fatores sociais, como grupos de referência, família, papéis sociais e status. Conforme Kotler (1998) salienta, todos os grupos têm influência direta ou indireta sobre atitudes ou comportamentos do consumidor. Além dos fatores culturais e sociais, há também as características pessoais que influenciam o comportamento do consumidor, tais como estilo de vida, estágio do ciclo de vida, personalidade e autoestima.

No entanto, o comportamento do consumidor é um processo decisório. A decisão de compra é um processo coerente e estruturado de tomada de decisão, em que a demanda exerce um papel importante no comportamento do consumidor. Isto é, o consumidor é influenciado, de modo que sua decisão de compra se desenvolve por meio de um processo coerente, afetando sua percepção e sua escolha em relação à relevância e à adequação dos produtos (SAMARA, MORSCH, 2005).

De acordo com Kotler e Armstrong (2007), o processo de decisão de compra é constituído de cinco estágios: 1) reconhecimento da necessidade - o comprador identifica um problema ou uma necessidade; 2) busca por informações - o consumidor busca informações para satisfazer necessidades; 3) avaliação das alternativas - são percebidos os riscos e os benefícios de cada opção; 4) decisão de compra - é a decisão de onde e quando realizar a compra; 5) comportamento pós-compra - após a compra, o comprador fica satisfeito ou insatisfeito.

Ressalta-se, contudo, que o estudo do comportamento do consumidor não consiste apenas em processos envolvidos na aquisição de bens e serviços por parte de indivíduos, denominados consumidores finais, mas abrange também os processos de compra de bens, serviços, 
matérias-primas ou insumos integrantes da cadeia de produção de determinados bens ou serviços, empreendidos por clientes institucionais e organizacionais, denominados consumidores organizacionais (PINHEIRO et al., 2011).

Em pleno século XXI, o consumidor assume um papel mais que consciente, sobretudo, como agente de variação da qualidade das relações de consumo e como influenciador no comportamento das organizações e instituições. Já o novo tipo de consumidor é um indivíduo politizado, consciente, bem informado e ético (SAMARA, MORSCH, 2005).

De acordo com os autores supracitados, conclui-se que compreender o consumidor é uma função essencial do marketing. Isso se torna relativamente importante na medida em que se identifica que o consumidor possui importante papel no cumprimento integral dos objetivos de uma organização, no processo de desenvolvimento dos produtos, assim como na decisão da produção e na disposição de bens e serviços no mercado.

\subsection{Satisfação do consumidor}

Todo o esforço mercadológico promovido pelas empresas gira em torno da satisfação do consumidor. As organizações visam necessariamente à satisfação das necessidades ou dos desejos dos consumidores, recebendo em troca o lucro almejado para a sustentabilidade do negócio (SAMARA, MORSCH, 2005).

Kotler (1998) define a satisfação como um sentimento de prazer ou de desapontamento, de acordo com o resultado desempenhado ou esperado pelo produto em relação às expectativas. Já para Blackwell et al. (2005), a satisfação é definida como a resposta do consumidor a uma avaliação da conexão percebida entre as expectativas e a performance após o consumo de um produto/serviço.
Para Spina, Giraldi e Oliveira (2013), a satisfação é o resultado da divergência entre as expectativas e o desempenho de um produto, percebido após o uso. A qualidade dos serviços é antecedente à satisfação do cliente; o cliente satisfeito tem intensão de recompra, não buscando, assim, a concorrência.

A busca pela satisfação do consumidor provém da adequação do que é oferecido pela empresa ao que os consumidores desejam ou necessitam. Porém, cada vez mais, torna-se difícil lidar com a fidelização de clientes dentre das várias opções de compras oferecidas ao consumidor, e, ao mesmo tempo que um consumidor volta para o local de compra, ele deve estar satisfeito com a transação (ARAÚJO, MIRANDA JUNIOR, 2011).

Samara e Morsch (2005) explicam que a satisfação aparece no comportamento pós-compra do consumidor; as sensações e atitudes determinarão se haverá novas compras e continuidade do relacionamento com a organização. Isto é, a avaliação pós-compra faz com que se perceba a existência de satisfação ou insatisfação em relação à compra: quando há insatisfação, o consumidor decide não confirmar sua escolha e não realizar novas compras ou conclui que a decisão tomada foi insensata e não volta a comprar o mesmo produto (marca ou modelo).

Por isso, de acordo com Kotler (1998), as empresas tendem à alta satisfação, pois, se o consumidor estiver apenas satisfeito, estará disposto a mudar quando surgir uma oferta melhor. Os consumidores plenamente satisfeitos estão menos dispostos a mudar, e a alta satisfação cria afinidade emocional e preferência racional pela marca, gerando, assim, lealdade por parte do consumidor.

Nesse sentido, convém destacar três tipos de satisfação que o consumidor espera em relação a determinado produto ou serviço: satisfação funcional, satisfação emocional e satisfação do benefício de uso. A satisfação funcional é obtida por meio de atributos 
tangíveis de um produto ou serviço que podem ser medidos de algum modo padronizado. A satisfação emocional, também chamada de satisfação psicológica, é aquela fornecida por benefícios intangíveis e não mensuráveis por meio de um padrão. Já a satisfação de benefício de uso está associada ao valor ganho pela propriedade e ao uso de um bem e serviço (SAMARA, MORSCH, 2005).

Entretanto, é fundamental que o profissional de marketing avalie a satisfação do seu consumidor e os custos para isso, assim como quais são as oportunidades de melhoria detectadas em relação ao cliente (SAMARA, MORSCH, 2005). Isso leva a concluir que o consumidor do século XXI se tornou mais exigente, mais consciente e mais poderoso em suas relações de consumo, buscando a satisfação das necessidades emocionais como status, prestígio e conquista.

Ao considerar o que foi exposto, percebe-se que a satisfação dos consumidores é amplamente discutido, contando inclusive com vasta literatura sobre o tema. Por esse motivo, buscou-se realizar um levantamento acerca de alguns estudos recentes, a fim de compreender as abordagens utilizadas até o momento.

Nesse sentido, o estudo de Pereira (2012) teve como objetivo analisar a relação entre o cenário temático de um restaurante e a satisfação de seus consumidores. Foi realizada uma pesquisa descritiva do tipo survey com uma amostra de 252 clientes de um restaurante temático da cidade de Florianópolis. Os dados foram analisados com o auxílio da estatística descritiva e multivariada e a utilização de coeficiente de correlação e análise fatorial. Os resultados mostraram que existe associação entre o cenário temático e a satisfação do consumidor e que a ambiência se correlaciona positivamente com a satisfação.

Por sua vez, Sampaio (2012) deteve-se em analisar a relação entre atributos e valores percebida por consumidores de comida japonesa de Fortaleza. A pesquisa sustenta-se teoricamente em abordagens sobre o comportamento do consumidor e em valores e na Teoria da Cadeia de Meios e Fins (Means End Chain-MEC). Foram realizadas 30 entrevistas em profundidade com consumidores de comida japonesa da cidade de Fortaleza entre os meses de novembro de 2008 e abril de 2009. O resultado da pesquisa evidenciou, entre os consumidores, valores individualistas como: abertura, vida excitante, satisfação, prazer, felicidade, autoestima, relação afetiva e amizade (SAMPAIO, 2012).

Já o estudo de Constantin, Abicht e Ceolin (2013) buscou identificar o comportamento do consumidor em relação aos produtos da microcervejaria Heilige, situada em Santa Cruz do Sul, Rio Grande do Sul. Para atingir os objetivos desse estudo, foram buscadas informações sobre os aspectos considerados valiosos ao desenvolvimento de estratégias que pudessem resultar na fidelização e na conquista de clientes, como marketing de relacionamento, comportamento de compra e processo de decisão e fidelização.Essa pesquisa, de natureza qualitativa-quantitativa e de delineamento exploratório-descritivo, foi realizada em duas etapas: a primeira etapa foi exploratória e bibliográfica de natureza qualitativa; já a segunda, descritiva de natureza quantitativa. Os resultados indicaram índices elevados de satisfação dos consumidores em relação a vários quesitos investigados, tais como qualidade, atendimento, entrega e preço (CONSTANTIN, ABICHT, CEOLIN, 2013).

Nessa mesma perspectiva, Ribeiro, Monteiro e Martins (2014) buscaram entender o comportamento do consumidor em relação ao valor gerado pelas versões impressa e eletrônica de uma revista, com o propósito de compreender como a natureza da informação fornecida por esses produtos complementares contribui para a lealdade e a satisfação dos consumidores. Para isso, foi realizada uma pesquisa quantitativa, utilizando como unidade de análise 
uma revista do mercado brasileiro. Uma amostra composta de 487 assinantes da revista foi examinada, e os resultados preliminares mostram que a versão eletrônica da revista, isoladamente, ainda é um produto de baixo valor agregado para os leitores (RIBEIRO, MONTEIRO, MARTINS, 2014).

Por último, uma pesquisa realizada por Oliveira, Silva, Rodrigues e Lopes (2015) permitiu verificar um processo de compras no varejo; a qualidade percebida e a satisfação, segundo eles, podem estar diretamente e positivamente correlacionadas com a lealdade. $\mathrm{O}$ artigo utilizou-se de uma investigação quantitativa, por meio de um survey de corte transversal único. A amostra utilizada foi por conveniência. Para a análise dos dados, foi empregada a técnica estatística de Modelagem em Equações Estruturais (MEE) por meio do software SmartPLS. Dentre os resultados obtidos, foi possível constatar que a qualidade percebida pelo consumidor no varejo e sua satisfação possuem uma relação positiva e direta com a lealdade.

Dessa forma, conclui-se que as pesquisas recentemente realizadas sobre o tema da satisfação e o comportamento do consumidor são de extrema importância para as organizações. Afinal, cada organização procura compreender, analisar e obter resultados a partir daquilo que é proposto no mercado de trabalho. Na tentativa de contribuir com essa temática, são apresentados, na seção seguinte, os procedimentos metodológicos desta pesquisa.

\section{Metodologia}

Esta pesquisa parte de uma abordagem qualitativa, uma vez que analisa dados numéricos, por meio de uma visão interpretativista, com auxílio da análise de conteúdo. De acordo com Silva e Menezes (2001), a pesquisa qualitativa entende que há uma relação dinâmica entre o mundo real e o sujeito. Quanto aos objetivos, o estudo carac- teriza-se pelo caráter descritivo, o que, segundo Samara e Morsch (2005), consiste em uma pesquisa que procura descrever situações de mercado a partir de dados primários, obtidos originalmente por intermédio de entrevistas pessoais ou discussões em grupos, relacionando e confirmando as hipóteses levantadas na definição do problema da pesquisa.

Esta pesquisa, de abordagem exploratória e caráter descritivo, visa descrever as características das organizações e os fenômenos inerentes ao seu contexto, revelando seus princípios da bibliometria. Conforme Guedes e Borschiver (2005), esse tipo de pesquisa se destaca pelo caráter descritivo tendo em vista a adoção da abordagem bibliométrica, que consiste em uma junção de leis e princípios empíricos que proporcionam o estabelecimento dos fundamentos teóricos da Ciência da Informação, por meio da contagem de documentos.

A fim de atingir o objetivo do estudo, foi feita uma busca na base de dado Scientific Periodicals Electronic Library (SPELL) em fevereiro de 2016. Considerou-se para a pesquisa a busca pelo termo satisfação do consumidor, considerando-se sua aparição apenas no resumo dos trabalhos, totalizando assim 102 artigos encontrados. Logo após, procedeu-se a um recorte temporal: foram selecionados artigos completos e em língua portuguesa publicados entre os anos de 2010 e 2015. Selecionou-se este período visto que o estudo se preocupa em compreender estratégias e tendências de pesquisas mais recentes. Assim, foram analisados os 60 artigos encontrados após a realização dos filtros mencionados.

$\mathrm{Na}$ etapa do mapeamento, uma vez organizados, os 60 artigos selecionados foram submetidos a uma análise detalhada. Ao longo do processo de leitura e interpretação dos artigos, foram identificados alguns focos importantes de análise. Esses focos serviram de base para a elaboração de uma tabela via software Excel, padronizada, para a análise de conteúdo individual de cada artigo. 
A tabela padronizada contemplou os seguintes tópicos: publicação por autor e gênero (1), quantidade de Instituições de Ensino Superior (IES) (2), publicação por periódico (3), publicação por ano (4), construto e modelo (5) e método (6). Foram considerados todos os artigos que se encontravam completos.

Por fim, a análise dos dados foi desenvolvida por meio da técnica de análise de conteúdo, que, conformeBardin(2004), consiste em um conjunto de técnicas de análise das comunicações, visando obter indicadores que permitam a geração de conhecimentos relativos às condições de produção/recepção dessas mensagens. Após a apresentação dos aspectos metodológicos que nortearam o desenvolvimento desta pesquisa, foi realizada a análise bibliométrica sobre as publicações identificadas na coleta dos dados, cujos resultados são apresentados a seguir. A Figura 1 representa o passo a passo utilizado para a realização desta pesquisa.

Figura 1: Passo a passo da pesquisa

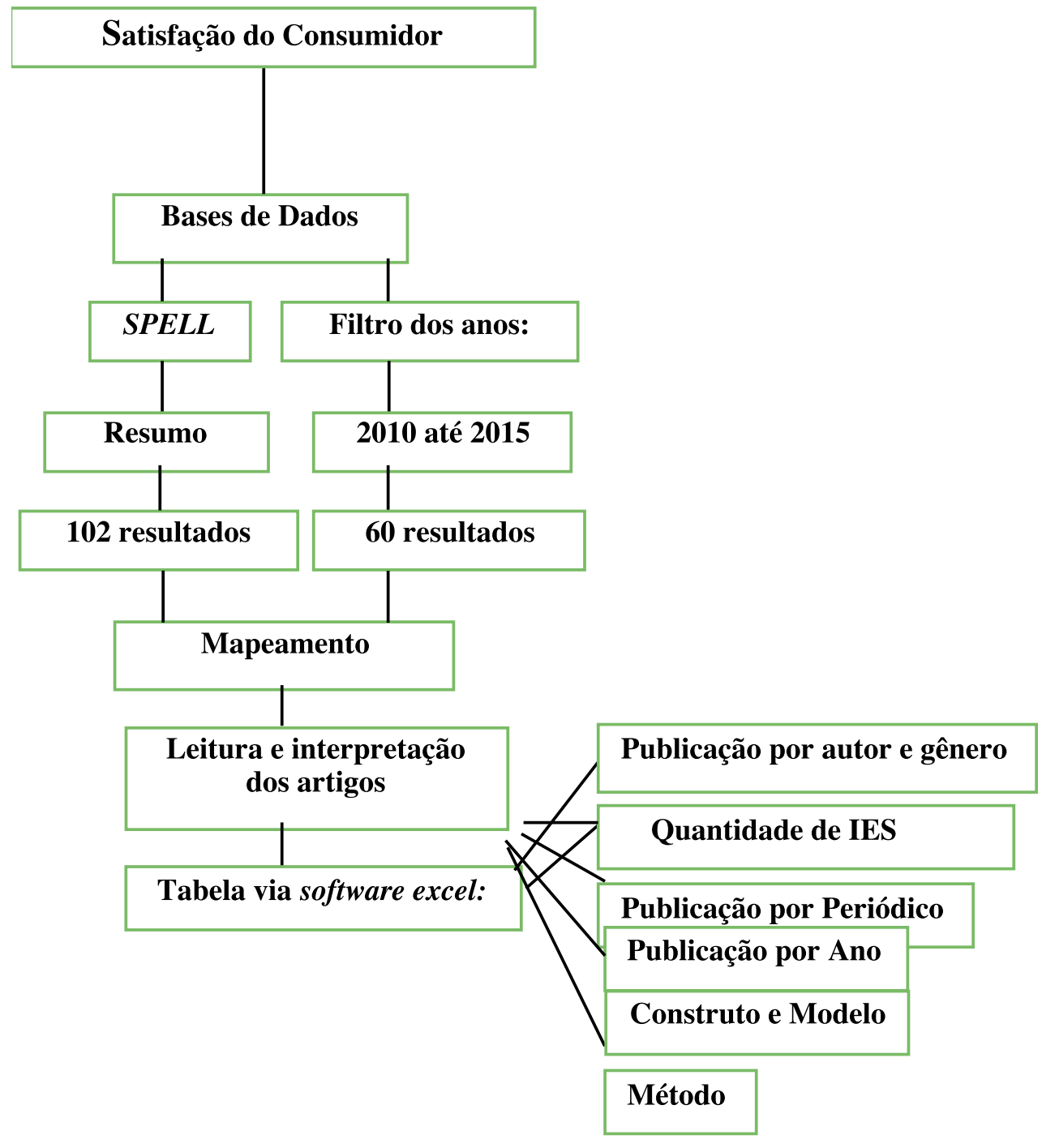

Fonte: Dados da pesquisa (2016) 


\section{Resultados e discussões}

Neste tópico, são discutidos os principais resultados encontrados a partir do desenvolvimento da pesquisa bibliométrica. A Tabela 1 apresenta uma síntese dos principais autores e suas publicações.

$\mathrm{Na}$ Tabela 1, é possível visualizar os autores que tiveram uma ou duas publicações. Os autores Mário $\mathrm{O}$.
Mota, Cláudio A. G. Nogueira, Felipe C. Almeida, Paulo G. N. Lima, Herber J. Moura, Paulo H. A. Curzio, Joyce G. Altaf, Irrene R. Troccolli e Eliane C. F. Maffezzolli tiveram duas publicações cada, ou seja, representaram $1 \%$ cada. No entanto, os demais autores somente apresentaram uma publicação sobre o tema, o que equivale a $91 \%$ dos 192 autores considerados.

Tabela 1: Quantidade de publicação por autor.

\begin{tabular}{c|c|c}
\hline AUTOR & $\begin{array}{c}\text { QUANT. DE PU- } \\
\text { BLICAÇ OES }\end{array}$ & $\%$ \\
\hline MÁRIO O. MOTA & 2 & $1 \%$ \\
\hline CLÁUDIO A. G. NOGUEIRA & 2 & $1 \%$ \\
\hline FELIPE C. ALMEIDA & 2 & $1 \%$ \\
\hline PAULO G. N. LIMA & 2 & $1 \%$ \\
\hline HERBER J. MOURA & 2 & $1 \%$ \\
\hline PAULO H. A. CURZIO & 2 & $1 \%$ \\
\hline JOYCE G. ALTAF & 2 & $1 \%$ \\
\hline IRRENE R. TROCCOLI & 2 & $1 \%$ \\
\hline ELIANE C. F. MAFFEZZOLLI & 2 & $1 \%$ \\
\hline DEMAIS AUTORES (1) & 174 & $91 \%$ \\
\hline TOTAL & $\mathbf{1 9 2}$ & $\mathbf{1 0 0}$ \\
\hline
\end{tabular}

Fonte: Dados da pesquisa (2016).

No entanto, outro procedimento realizado foi a visita aos currículos Lattes dos autores mais prolíficos para verificação de formação e área de atuação. Os autores Mário O. Mota, Cláudio A. G. Nogueira, Herber J. Moura, Paulo H. A. Curzio e Irrene R. Troccoll possuem experiência nas áreas de Administração e Economia e ênfase em Marketing. Somente o autor Paulo G. N. Lima, recentemente formado em Administração, atua principalmente na área de planejamento estratégico. Considerando esses dados, verificou-se que as principais atuações dos autores se referem aos seguintes temas: Comportamento do Consumidor, Marketing de Relacionamento, Métodos Quantitativos e Qualitativos Aplicados, Desenvolvimento Econômico, Estratégias Empresariais, Grupos Estratégicos e Redes de Relacionamento.

Em relação aos gêneros masculino e feminino, obtiveram-se os seguintes resultados: a maior quantidade de publicação foi do gênero masculino, com 106 pesquisadores, equivalente a $55 \%$ do total. Já em relação ao gênero feminino, contabilizaram-se 86 pesquisadoras, representando um total de $45 \%$. Na Tabela 2, são apresentadas as quantidades de publicações por IES.

$\mathrm{Na}$ Tabela 2, apresentam-se as dez IES que tiveram maior número de publicação sobre o tema satisfação do consumidor. Vale salientar que a pesquisa foi realizada a partir da análise de autores por publicação de cada universidade, obtendo assim um total de 171 publicações por IES. Esses dados apontam para duas variáveis importantes de análise: a primeira mostra a quantidade total de publicações de artigos por IES, enquanto a segunda apenas indica o valor percentual em relação à primeira - estabelecendo, dessa forma, a percentagem da participação dos artigos de satisfação do consumidor em relação ao total de artigos de cada IES. 
Tabela 2: Quantidade de Publicação de Autor por IES.

\begin{tabular}{|c|c|c|}
\hline IES & $\begin{array}{l}\text { QUANT. DE } \\
\text { PUBLIC. }\end{array}$ & $\%$ \\
\hline Universidade de Fortaleza (UNIFOR) - Brasil. & 17 & $10 \%$ \\
\hline $\begin{array}{l}\text { Universidade de São Paulo (USP) - Bra- } \\
\text { sil. }\end{array}$ & 9 & $5 \%$ \\
\hline $\begin{array}{c}\text { Universidade Federal do Rio Grande do } \\
\text { Sul (UFRGS) - Brasil. }\end{array}$ & 9 & $5 \%$ \\
\hline $\begin{array}{l}\text { Universidade Federal de Santa Maria } \\
\text { (UFSM) - Brasil. }\end{array}$ & 8 & $5 \%$ \\
\hline $\begin{array}{l}\text { Universidade Federal do Paraná (UFPR) } \\
\text { - Brasil. }\end{array}$ & 7 & $4 \%$ \\
\hline $\begin{array}{l}\text { Universidade Federal de Minas Gerais } \\
\text { (FUMG) - Brasil. }\end{array}$ & 6 & $4 \%$ \\
\hline $\begin{array}{l}\text { Universidade Federal de Pernambuco } \\
\text { (UPFE) - Brasil. }\end{array}$ & 6 & $4 \%$ \\
\hline $\begin{array}{l}\text { Faculdade de Tecnologia Senac Jaraguá } \\
\text { do Sul - Brasil. }\end{array}$ & 6 & $4 \%$ \\
\hline $\begin{array}{l}\text { Faculdade Integrada Vianna Júnior - } \\
\text { Brasil. }\end{array}$ & 5 & $3 \%$ \\
\hline $\begin{array}{l}\text { Universidade Metodista de Piracicaba } \\
\text { (UNIMEP) -Brasil. }\end{array}$ & 5 & $3 \%$ \\
\hline DEMAIS IES (4) & 36 & $21 \%$ \\
\hline DEMAIS IES (3) & 21 & $12 \%$ \\
\hline DEMAIS IES (2) & 14 & $8 \%$ \\
\hline DEMAIS IES (1) & 22 & $13 \%$ \\
\hline TOTAL & 171 & $100 \%$ \\
\hline
\end{tabular}

Fonte: Dados da pesquisa (2016).

Pela análise da Tabela 2, verifica-se que a Universidade de Fortaleza (UNIFOR) possui ao todo 17 publicações; isso se deve ao fato de a Instituição contar com um Núcleo de Estudos Internacionais (NEI) com o intuito de estudar diversos países e continentes nas áreas de Comércio Internacional, Negócios e também Marketing. Com nove publicações, consta a Universidade de São Paulo (USP), que possui ajuda de Núcleos de Apoio à Pesquisa (NAPs), Centros de Pesquisa, Inovação e Difusão (CEPIDs) e Grupos de Pesquisas sobre diversas áreas de conhecimento. Já a Universidade Federal de Santa Maria (UFSM), mesmo sem ter um grupo de estudos destinado ao assunto, , possui vários autores que estão pesquisando o tema. Em seguida, aparece a Universidade Federal do Paraná (UFPR), que também não possui grupo de estudos específico sobre o tema, mas tem uma importante pesquisadora, a autora Eliane Cristine Francisco Maffezzolli.

As Universidades Federais de Minas Gerais (UFMG) e de Pernambuco (UFPE) e a Faculdade de Tecnologia Senac Jaraguá do Sul apresentaram, juntas, 18 publicações, representando $4 \%$ cada. Já a Faculdade Integrada Vianna Júnior e a Universidade Metodista de Piracicaba tiveram, juntas, dez publicações. As demais instituições, com quatro e três publicações, totalizaram 57 publicações. E, por último, as instituições com uma ou duas publicações apresentaram 36 publicações. A seguir, a Tabela 3 demonstra as principais publicações por periódico por ano. 
Tabela 3: Quantidade de Publicação por Periódico.

\begin{tabular}{c|c|c|c|c|c|c|c|c}
\hline PERÍODICO & 2010 & 2011 & 2012 & 2013 & 2014 & 2015 & QUANT. & $\%$ \\
\hline $\begin{array}{c}\text { REMark - Revista Brasileira } \\
\text { de Marketing }\end{array}$ & & & 2 & 2 & 2 & 2 & 8 & $13 \%$ \\
\hline Revista Alcance - Eletrônica & 2 & & 2 & & & & 4 & $7 \%$ \\
\hline $\begin{array}{c}\text { BASE - Revista de Admi- } \\
\text { nistração e Contabilidade da } \\
\text { Unisinos }\end{array}$ & & 2 & & & & 2 & $3 \%$ \\
\hline $\begin{array}{c}\text { RAE - Revista de Adminis- } \\
\text { tração }\end{array}$ & & 2 & & & & & 2 & $3 \%$ \\
\hline DEMAIS PERIÓDICOS (1) & 6 & 4 & 8 & 9 & 11 & 6 & 44 & $73 \%$ \\
\hline TOTAL & 8 & 6 & 14 & 11 & 13 & 8 & 60 & $100 \%$ \\
\hline
\end{tabular}

Fonte: Dados da pesquisa (2016).

Desse modo, com o intuito de identificar quais são os principais periódicos que publicam artigos sobre satisfação do consumidor, foram sintetizados, na Tabela 3, os nomes dos periódicos, a quantidade de publicação e sua respectiva porcentagem.

Após a análise, pode-se perceber que o periódico REMark - Revista Brasileira de Marketing apresentou o maior número de publicações sobre o tema, com oito trabalhos entre os anos 2012, 2013, 2014 e 2015, seguido pelo periódico Revista Alcance - Eletrônica, com quatro arti- gos publicados entre os anos 2010 e 2012 . Percebe-se, ainda, grande interesse em publicações sobre satisfação do consumidor nos periódicos relacionados à satisfação $\mathrm{e}$ ao comportamento do consumidor.

Essa análise dos principais periódicos que publicaram artigos sobre $o$ tema fornece importantes informações para fins de análise e indica para onde devem ser enviados artigos acerca do tema satisfação do consumidor.

O Gráfico 1, exposto a seguir, apresenta a quantidade de publicação de acordo com a evolução no decorrer dos anos.

\section{Gráfico 1: Quantidade de Publicação por Ano.}

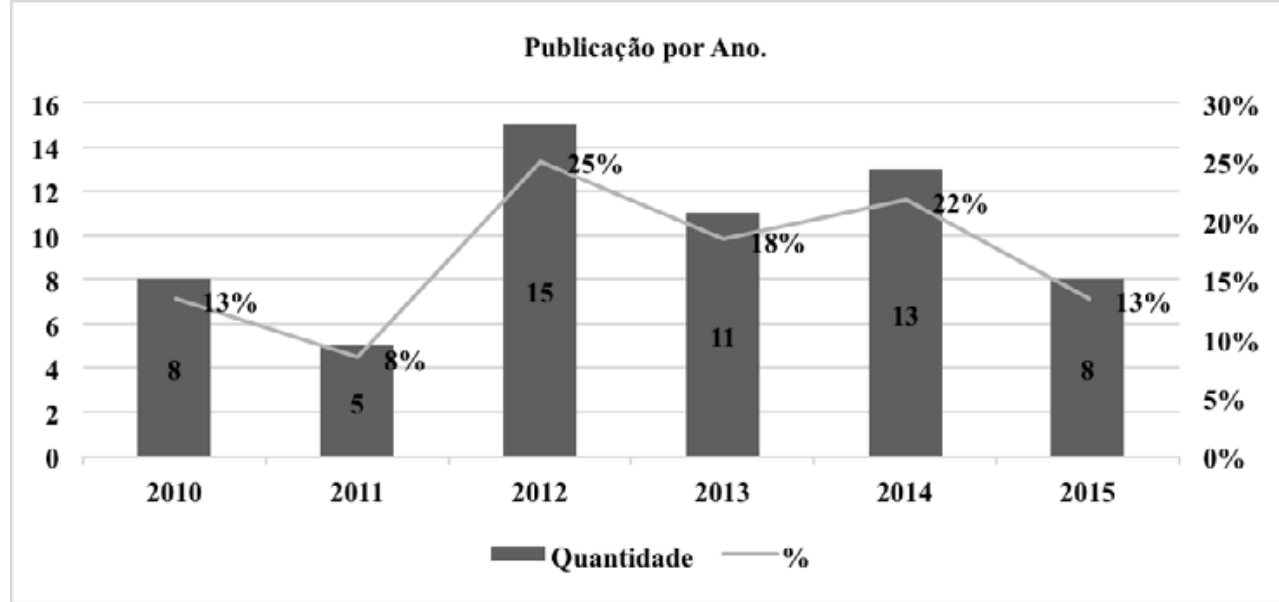

Fonte: Dados da pesquisa (2016). 
No Gráfico 1, é possível observar que os anos de 2012, 2013 e 2014 foram os que tiveram o maior número de publicações relacionadas ao tema, totalizando, especificamente neste período, 39 artigos. Dessa forma, pode-se afirmar que a maior parte das publicações referentes ao tema se concentra a partir do ano de 2012 $(25 \%)$. Ou seja, a partir desse ano, os pes- quisadores tiveram uma atuação abrangente na realização de pesquisas sobre a satisfação do consumidor, aplicando suas pesquisas em diversos setores, como turismo, hotéis e restaurantes, por exemplo.

A seguir, são apresentados, na Tabela 4, os principais constructos utilizados nas pesquisas relacionadas à satisfação do consumidor.

Tabela 4: Construtos mais utilizados.

\begin{tabular}{c|c|c}
\hline CONSTRUTOS & QUANTIDADE & \% \\
\hline Comportamento do Consumidor & 8 & $13 \%$ \\
\hline Lealdade & 6 & $10 \%$ \\
\hline Valor percebido & 6 & $10 \%$ \\
\hline Comprometimento do Consumidor & 5 & $8 \%$ \\
\hline Confiança & 4 & $7 \%$ \\
\hline Qualidade de serviços & 4 & $7 \%$ \\
\hline Varejo de Serviços & 4 & $7 \%$ \\
\hline Qualidade percebida & 3 & $5 \%$ \\
\hline Paradigma da Desconformidade & 3 & $5 \%$ \\
\hline Marketing de Relacionamento & 2 & $3 \%$ \\
\hline TOTAL & $\mathbf{6 0}$ & $\mathbf{1 0 0 \%}$ \\
\hline
\end{tabular}

Fonte: Dados da Pesquisa (2016).

Ao analisar os artigos selecionados neste estudo bibliométrico, identificou-se que os autores relacionaram uma série de construtos; dentre eles, vale destacar: comportamento do consumidor, lealdade, comprometimento, confiança, qualidade percebida e marketing de relacionamento. $\mathrm{O}$ construto mais utilizado em relação ao tema satisfação do consumidor foi o de comportamento do consumidor, com oito publicações, equivalendo a $13 \%$ do total.

O construto lealdade, conforme Oliver (1997), refere-se à razão da probabilidade de recompra e à razão da troca de fornecedor pela qualidade e pelo atendimento nos serviços prestados. Segundo Mowen e Minor (2003, p. 442), o comprometimento é o principal antecedente da lealdade, pois esta só é adquirida quando o consumidor apresenta uma atitude positiva e percebe um compromisso mais forte em relação à empresa.
Muitos autores afirmam que a lealdade é influenciada por outros precedentes, como a confiança, o valor percebido e o próprio comprometimento.

De acordo com Johnson et al., (2001), o valorpercebidoéigualado ao desempenho percebido da qualidade recebida em relação ao preço pago. Portanto, ao adquirir um produto ou serviço, o consumidor interessa-se em compará-lo com outros itens equivalentes no que concerne ao valor.

O construto confiança é citado na literatura como um forte influenciador do comprometimento, sendo sinônimo de garantia quanto ao desempenho consistente e competente da empresa, garantindo a segurança e o menor risco da compra, além de reduzir as incertezas (MORGAN; HUNT, 1994).

Outro construto utilizado foi o paradigma da desconformidade apresentado por Oliver (1980). Nesse pa- 
radigma, idealiza-se a satisfação e a insatisfação como o resultado de uma comparação entre as expectativas anteriores à compra/consumo e o desempenho percebido pelo consumidor.

Considerando os construtos analisados na Tabela 5, pode-se afirmar principalmente que o marketing de relacionamento é a forma pela qual a empresa encontra o cliente, passa a conhecê-lo e mantém contato com ele, buscando assegurar o cumprimento das expectativas do cliente com relação ao produto, ao serviço, à qualidade, à entrega, à comunicação, ao pós-venda, entre outras variáveis (CONSTANTIN; ABICHT; CEOLIN, 2013).

Na sequência, a Tabela 5 apresenta uma síntese dos autores dos modelos mais utilizados nas pesquisas em análise.

Tabela 5: Autor do modelo utilizado na pesquisa.

\begin{tabular}{c|c|c}
\hline AUTORES & QUANTIDADE & \% \\
\hline OLIVER (1997) & 8 & $13 \%$ \\
\hline OLIVER (1999) & 4 & $7 \%$ \\
\hline MARCHETTI E PRADO (2001) & 4 & $7 \%$ \\
\hline OLIVER (1980) & 3 & $5 \%$ \\
\hline OLIVER (1981) & 2 & $3 \%$ \\
\hline OLIVER (1993) & 2 & $3 \%$ \\
\hline FORNELL (2006) & 2 & $3 \%$ \\
\hline SAMARA E MORSCH (2005) & 2 & $3 \%$ \\
\hline DEMAIS AUTORES (2) & 8 & $13 \%$ \\
\hline DEMAIS AUTORES (1) & 25 & $42 \%$ \\
\hline TOTAL & $\mathbf{6 0}$ & $\mathbf{1 0 0 \%}$ \\
\hline
\end{tabular}

Fonte: Dados da Pesquisa (2016).

Diante dos resultados expostos, identificou-se que o autor Oliver (1997) foi o que mais se destacou, representando $13 \%$ dos modelos. Em seguida, estão os autores Marchetti e Prado (2001), com quatro publicações, equivalendo a $7 \%$. Já os demais autores apresentam uma ou duas quantidades de modelos utilizados.

Frente a esses dados, percebe-se que o autor Oliver $(1980 ; 1981 ; 1993$; 1997; 1999) mantém um foco especial sobre a satisfação do consumidor, a fidelização de clientes e os processos de pós-compra em geral.
Cabe destacar, ainda, que, apesar de vários estudos a respeito de satisfação dos consumidores já terem sido desenvolvidos, Marchetti e Prado (2004) afirmam que novos estudos são essenciais para avançar no tema e obter resultados mais verdadeiros a respeito da satisfação dos consumidores por meio de indicadores técnicos de desempenho e da avaliação dos serviços prestados pelas empresas.

Por fim, na Tabela 6 apresentamse as principais abordagens de pesquisas empregadas nos estudos sobre satisfação do consumidor.

Tabela 6: Abordagens de pesquisa.

\begin{tabular}{c|c|c}
\hline Abordagem & Quantidade & $\%$ \\
\hline Quantitativa & 34 & $57 \%$ \\
\hline Qualitativa & 11 & $18 \%$ \\
\hline Quali e Quanti & 9 & $15 \%$ \\
\hline Revisão Teórica & 6 & $10 \%$ \\
\hline Total & $\mathbf{6 0}$ & $\mathbf{1 0 0 \%}$ \\
\hline
\end{tabular}

Fonte: Dados da Pesquisa (2016). 
Ao analisar a metodologia utilizada pelos autores dos artigos, verificou-se uma maior incidência para os estudos de natureza quantitativa, totalizando 34 artigos, o que representa um percentual de $55 \%$. A pesquisa quantitativa considera que tudo pode ser quantificável e requer o uso de recursos e de técnicas estatísticas, como percentagem e média (SILVA; MENEZES, 2001).

A abordagem qualitativa apresentou um percentual de $18 \%$, ou seja, 11 artigos optaram por realizar suas pesquisas por meio de entrevistas. A abordagem mista de pesquisa, quali-quantitativa, de delineamento exploratório e descritivo também foi apresentada nos artigos analisados, representando $15 \%$ do total.

Já o método de revisão teórica também se faz presente nos artigos encontrados sobre satisfação do consumidor, totalizando seis artigos, que consistem em artigos somente teóricos, sem nenhuma intenção de aplicação de pesquisas.

\section{Considerações finais}

Com o intuito de explorar o tema satisfação do consumidor, diversas análises foram realizadas no decorrer deste artigo a partir do método de pesquisa bibliométrica, com o auxílio do software Excel. Ao analisar 60 artigos publicados em periódicos nacionais no período que compreendeu os anos de 2010 a 2015 , foi possível identificar algumas características inerentes ao tema.

A partir dos resultados encontrados, evidencia-se que as pesquisas realizadas sobre o tema vêm aumentando no decorrer dos anos e também que há IES que possuem interesse em publicações sobre satisfação do consumidor. Isso demonstra que o tema ainda tem muito a crescer em termos de pesquisa, permitindo assim que novos pesquisadores busquem preencher as lacunas existentes.

É possível identificar também a evolução do número de trabalhos publicados por esses autores em periódicos nacionais. Dentre os periódicos, a RE-
Mark - Revista Brasileira de Marketing apresentou o maior número de publicações sobre o tema, seguida do periódico Revista Alcance - Eletrônica. Esse resultado sugere que estes periódicos possuem interesse em pesquisas relacionadas ao tema satisfação do consumidor, induzindo que futuras pesquisas sejam direcionadas para estas revistas.

Os anos de 2012, 2013 e 2014 destacam-se pelo número expressivo de artigos publicados sobre o tema; contudo, a quantidade de artigos encontrados em cada um dos anos foi de 15, 11 e 13, respectivamente. Os resultados em relação ao aumento da pesquisa a cada ano indicam que, a partir de 2012, o tema começou a ser alvo de maior interesse entre os pesquisadores. Já em 2015, houve somente oito publicações sobre o tema. O tema é crescente, pois sua importância vem merecendo um grande número de publicações; todavia, sugere-se que seja aumentada a quantidade de pesquisas que abordem o tema satisfação do consumidor nos próximos anos.

Dentre as variáveis para a mensuração da satisfação, torna-se evidente a maior utilização das medidas desenvolvidas por Oliver (1997, p.13), que define a satisfação como a resposta à completude do consumidor. Ou seja, trata-se da análise da qualidade do produto/serviço, verificando se o produto/ serviço em si ofereceu um nível prazeroso de completude relativo ao consumo, incluindo níveis maiores ou menores de completude, com a utilização de medidas voltadas para o comportamento e à satisfação, que foi a escala mais utilizada nas pesquisas analisadas.

Em relação às abordagens de pesquisa empregadas pelos autores, as pesquisas quantitativas do tipo survey tiveram maior destaque, representando $57 \%$ do total dos artigos analisados. A análise dos artigos sugere também que mais pesquisas longitudinais, exploratórias e qualitativas sejam futuramente realizadas por pesquisadores. Ainda, identificaram-se lacunas de pesquisa no que tange a uma 
quantidade maior de estudos desenvolvidos em países emergentes, em micro e pequenas empresas e em outros setores, principalmente no setor industrial.

Foi possível evidenciar, assim, que ainda existe uma tendência de estudos com viés positivista, de abordagem quantitativa, nas ciências sociais aplicadas e principalmente nos estudos da área da administração. Os estudos qualitativos estão crescendo, mesmo que de forma incipiente. Acredita-se que a área de marketing seja uma das áreas que precisa buscar diferentes estratégias de pesquisa, a fim de encontrar soluções para os novos problemas que são enfrentados no século XXI.

Percebe-se, a partir dos resultados apresentados, que o tema é de crescente interesse na academia nos últimos anos e que diversas pesquisas têm utilizado o termo satisfação do consumidor ou satisfação do cliente. Pode-se considerar que os trabalhos encontrados até o momento são apenas o princípio de uma construção mais profunda que precisa ser desenvolvida sobre a temática, considerando que as variáveis para mensuração da satisfação do consumidor precisam ser mais bem desenvolvidas e podem contribuir para a efetivação da satisfação nas organizações.

Uma das limitações desta pesquisa provém da base de dados utilizada, pois esta pode estar limitada a apenas alguns periódicos. Outra limitação se deve aos filtros metodológicos empregados, que foram definidos para pesquisar o termo satisfação do consumidor por título e resumo, o que pode ocasionar uma boa pesquisa, com exceção de alguns artigos irrelevantes relacionados ao tema.

Futuras pesquisas precisam direcionar esforços para a ampliação deste estudo, que pode ser analisado à luz de outras variáveis que não foram aqui abordadas, como, por exemplo, temas de pesquisas relacionados com mais concretude à satisfação do consumidor ou, ainda, à origem dos pesquisadores. Também existe a importância e a necessidade de realizar uma pesquisa como esta em bases de dados internacionais, identificando, assim, como está o estado da arte da pesquisa em satisfação no Brasil.

Como mencionado pelos autores dos artigos analisados, o tema satisfação do consumidor é revolucionário e ainda merece ser desenvolvido. A importância da realização de futuras pesquisas em outras empresas, de diferentes setores, principalmente no setor de indústrias e também em micro e pequenas empresas, é pertinente.

\section{Referências}

ARAÚJO, G. C.; MIRANDA J. L. J. Satisfação dos consumidores que frequentam bares. Revista Hospitalidade, v. 8, n. 2, p. 121-146, 2011.

BARDIN, L. Análise de conteúdo. 3. ed. Lisboa: Edições 70, 2004.

BLACKWELL, R.D., MIRINIARD, P.W., ENGEL, J.F. Comportamento do consumidor. São Paulo: Pioneira Thomson Learning, 2005

CONSTANTIN, H.; ABICHT, A. M.; CEOLIN, A. C. Comportamento do consumidor: produtos de uma micro cervejaria. Desafio Online, v. 1, n. 1, p. 1-22, 2013.

ENGEL, J. F.; BLACKWELL, R. D.; MINIARD, P. W. Comportamento do consumidor. Rio de Janeiro: LTC, 2000.

JOHNSON, M. D., GUSTAFSSON, A. A. T. W., LERVIK, L. C. J. The evolution and future of national customer satisfaction index models. Journal of Economic Psychology, v.22, p.217 - 245, 2001.

GUEDES, V. L. S.; BORSCHIVER, S. Bibliometria: uma ferramenta estatística para a gestão da informação e do conhecimento, em sistemas de informação, de comunicação e

de avaliação científica e tecnológica. 2005. Disponível em: <http://dici.ibict. br/archive/00000508/>. Acesso em: 19/12/2016. 
KOTLER, P. Administração de marketing: análise, planejamento, implementação e controle (Tradução: Airton Bomfim Brandão). 5ªd. São Paulo: Atlas, 1998.

KOTLER, P. Administração de marketing: a edição do novo milênio. 10 . ed. São Paulo: Prentice Hall, 2000.

KOTLER, P.; ARMSTRONG, G. Princípios de Marketing. Tradução de Cristina Yamagami. Rio de Janeiro: Prentice Hall, 2007.

LEITE, R.S.; GONÇALVES FILHO, C. Um estudo empírico da aplicação do índice europeu de satisfação do cliente (ECSI) no Brasil. Revista de Administração Mackenzie. v. 8, n 4, p. 178200, 2007.

MARCHETTI， R.; PRADO, P.H.M. Avaliação da satisfação do consumidor utilizando o método de equações estruturais: um modelo aplicado ao setor elétrico brasileiro. Revista de Administração Contemporânea, v. 8, n. 4, p. 09-32, 2004.

MORGAN, R. M.; HUNT, S. D. The commitmenttrust theory of relationship marketing. Journal of Marketing, v. 58, p. 20-38, Jul. 1994.

MOWEN, J. C.; MINOR, M. Comportamento do consumidor. São Paulo: Makron Books, 2003.

OLIVEIRA, A. S.; SILVA, D.; LOPES, E. L.; RODRIGUES, G. V. A Qualidade Percebida pode ser um Antecedente Direto da Lealdade assim Como a Satisfação? Um Estudo em Processo de Compra no Varejo. Revista Gestão \& Planejamento, v. 16, n. 2, p. 134-148, 2015.

OLIVER, R. L. A cognitive model of antecedentes and consequences of satisfaction decisions. Journal of Marketing Research, v. 17, p. 460-469, 1980.

OLIVER, R. L. Effect of expectations and disconfirmation on postexposure product evaluations: an alternative interpretation. Journal of Applied Psychology, v. 62, n.4, p. 480-486, 1997.
PEREIRA, E. W. Cenário temático de restaurante e a satisfação do consumidor. Revista de Administração FACES Journal, v. 11, n. 4, p. 149-176, 2012.

PINHEIRO, R. M; CASTRO, G. C; SILVA, H. H; NUNES, J. M. G. Comportamento do consumidor. $1^{\mathrm{a}} \mathrm{Ed}$. Rio de Janeiro: Editora FGV, 2011.

RIBEIRO. H. P.; MONTEIRO, P. R. R.; MARTINS, F. D. Mídia impressa e eletrônica: uma análise sob a ótica do valor para o cliente na gestão de produtos. Revista de Administração e Inovação, v. 11, n. 1, p. 6-28, 2014.

SAMARA, B. S.; MORSCH, M. A. Comportamento do consumidor: conceitos e casos. São Paulo: Prentice Hall, 2005.

SAMPAIO, D. O. et al. Um estudo comparativo sobre o comportamento do consumidor de automóveis novos. 2004. Disponível em: <http://www. ead.fea.usp.br/ semead/7semead/paginas/artigos\%20recebidos/marketing/ MKT76 Um Estudo comparativo do comp consumid.PDF $>$. Acesso em: $17 \overline{\text { de }}$ abrì de 2016.

SAMPAIO, J. A. C. Amizade, prazer e felicidade: relações entre atributos e valores na ótica de consumidores de comida japonesa. Contextus - Revista Contemporânea de Economia e Gestão, v. 10, n. 1, p. 67-82, 2012.

SILVA, E. L; MENEZES, E. M. Metodologia da pesquisa e elaboração de dissertação. 3. ed. Rev. Atual. Laboratório de Ensino a Distância Da UFSC. Florianópolis, 2001.

SPINA, D. T.; GIRALDI, J. M. E.; OLIVEIRA, M. M. B. A influência das dimensões da qualidade de serviços na satisfação do cliente: um estudo em uma empresa do setor de controle de pragas. Revista de Gestão, v. 20, n. 1, p. 93$112,2013$. 